\title{
STRUCTURAL STUDY BY RIETVELD REFINAMENT AND PHOTOLUMINESCENT INVESTIGATION OF Ba(Mo1-xWx)04 CRYSTALS
}

Nogueira, I. C. ${ }^{1}{ }^{*}$; de Jesus, M. M. ${ }^{1}$; Gonçalves, R. F. ${ }^{2}$; Pereira, P.F.S. ${ }^{3}$; Longo, E. ${ }^{4}$; Rivas Mercury, J. M. ${ }^{1}$

${ }^{1}$ Programa de Pós-Graduação em Engenharia de Materiais, Instituto Federal de Educação, Ciência e Tecnologia do Maranhão, São Luís, MA, Brazil

${ }^{2}$ Departamento de Química, Universidade Federal de São Paulo, São Paulo, SP, Brazil

${ }^{3}$ Departamento de Química, Universidade Federal de São Carlos, São Carlos, SP, Brazil

${ }^{4}$ Instituto de Química, Universidade Estadual Paulista, Araraquara, SP, Brazil

*isamiracosta@gmail.com

In this work, molybdate tungstate of barium $\mathrm{Ba}\left(\mathrm{Mo}_{1-x} \mathrm{~W}_{x}\right) \mathrm{O}_{4}(x=0,0.25,0.50,0.75$ and 1$)$ crystals were prepared by co-precipitation method. Detailed crystal structure studies were performed using X-ray diffraction data obtained at room temperature. The application of the Rietveld method confirmed the reported tetragonal $I 4_{1} /$ a phase. Changes in the lattice parameters, unit-cell volume and polyhedral distortions in the compounds are explained as a function of $(x)$ composition. The shape and size of the crystals were observed by means of field emission scanning electron microscopy (FE-SEM). The FE-SEM images indicate that increased $(x)$ content produces a decrease in the crystal size. The optical properties were investigated by ultraviolet-visible (UV-Vis) absorption spectroscopy and photoluminescence (PL) measurements. Photoluminescent emission at room temperature with different maximum intensity was assigned to different densities of intermediate electron levels (deep and shallow) located within the band gap. 